\title{
AKARI NIR spectroscopy of interstellar ices
}

\section{Takashi Onaka ${ }^{1}$, Tamami I. Mori ${ }^{1}$, Itsuki Sakon ${ }^{1}$, Fumihiko Usui ${ }^{1}$, Ronin $\mathbf{W u}^{1}$ and Takashi Shimonishi ${ }^{2}$}

\author{
${ }^{1}$ Department of Astronomy, Graduate School of Science, The University of Tokyo \\ 7-3-1 Hongo, Bunkyo-ku, Tokyo 113-0033, Japan \\ email: onaka@astron.s.u-tokyo.ac.jp \\ ${ }^{2}$ Frontier Research Institute for Interdisciplinary Sciences, Tohoku University \\ 6-3 Aramakiazaaoba, Aoba-ku, Sendai, Miyagi, 980-8578, Japan
}

\begin{abstract}
The Infrared Camera (IRC) onboard $A K A R I$ has a near-infrared $(2-5 \mu \mathrm{m})$ spectroscopic capability with high sensitivity that allows us to study the major ice components in various objects. In particular, $\mathrm{H}_{2} \mathrm{O}$ and $\mathrm{CO}_{2}$ ice absorption features have been detected towards nearby galaxies, including several young stellar objects (YSOs) in the Large Magellanic Cloud (LMC), as well as a number of HII region-PDR complexes for the first time by IRC spectroscopy. While observations in the LMC show a high ratio $(\sim 0.34)$ of the $\mathrm{CO}_{2}$ to $\mathrm{H}_{2} \mathrm{O}$ ice column densities, the ratios in Galactic HII-region-PDR complexes are in the range of $0.1-0.2$, being compatible with those found in Galactic massive YSOs in previous studies. The good correlation supports concurrent formation of the two ice species on the grain surface and the higher ratio in the low-metallicity LMC suggests possible environmental effects in the formation process.
\end{abstract}

Keywords. (ISM:) dust, extinction, ISM: lines and bands, infrared: ISM, astrochemistry

Ices of various species play important roles in interstellar chemistry (e.g., van Dishoeck 2014). Characteristic bands of ice species reside in the near- to mid-infrared $(2-20 \mu \mathrm{m})$ and thus infrared observations are the most efficient means for the study of ices in the interstellar medium (ISM). ISO and Spitzer studied ice features towards deeply embedded young stellar objects (YSOs) and stars behind molecular clouds, making significant progress in our understanding of interstellar ices (e.g., Gibb et al. 2004; Pontoppidan et al. 2008). They indicate good correlations between the column densities of $\mathrm{H}_{2} \mathrm{O}$ and $\mathrm{CO}_{2}$ ices, suggesting concurrent formation of the two ice species on the grain surface (Ippolo et al. 2011; Oba et al. 2012).

The Infrared Camera (IRC) onboard $A K A R I$ offered high-sensitivity spectroscopy in 2.5-5 $\mu \mathrm{m}$ (Onaka et al. 2007), where major ice species have strong absorption bands, such as $\mathrm{H}_{2} \mathrm{O}$ at $3.05 \mu \mathrm{m}, \mathrm{CO}_{2}$ at $4.27 \mu \mathrm{m}, \mathrm{XCN}$ at $4.62 \mu \mathrm{m}$, and $\mathrm{CO}$ at $4.67 \mu \mathrm{m}$. The IRC allows us to study ice absorption features in faint objects, including nearby galaxies and relatively evolved diffuse objects. A recent study of ices in nearby galaxies with the IRC suggests no clear correlation between $\mathrm{H}_{2} \mathrm{O}$ and $\mathrm{CO}_{2}$ ices, which may be attributed to the relatively large beam $\left(\sim 5^{\prime \prime}\right)$ that contains various components on the line-of-sight (Yamagishi et at. 2015). Studies of ice absorption towards YSOs in the Large Magellanic Cloud (LMC) with the IRC, on the other hand, show by a factor of 2 larger column densities of $\mathrm{CO}_{2}$ ice relative to $\mathrm{H}_{2} \mathrm{O}$ ice than towards Galactic massive YSOs, which must be related to the low-metallicity condition of the LMC and give important implication on the ice formation process (Shimonishi et al. 2008, 2010). A study of Galactic molecular clouds with the IRC further suggests multi-stage ice formation (Noble et al. 2013).

In this report, we present latest results of NIR spectroscopy of ice species in Galactic HII-photodissociation region (PDR) complexes made with the IRC. Those targets are extended and relatively evolved compared to YSOs in previous studies and thus may 

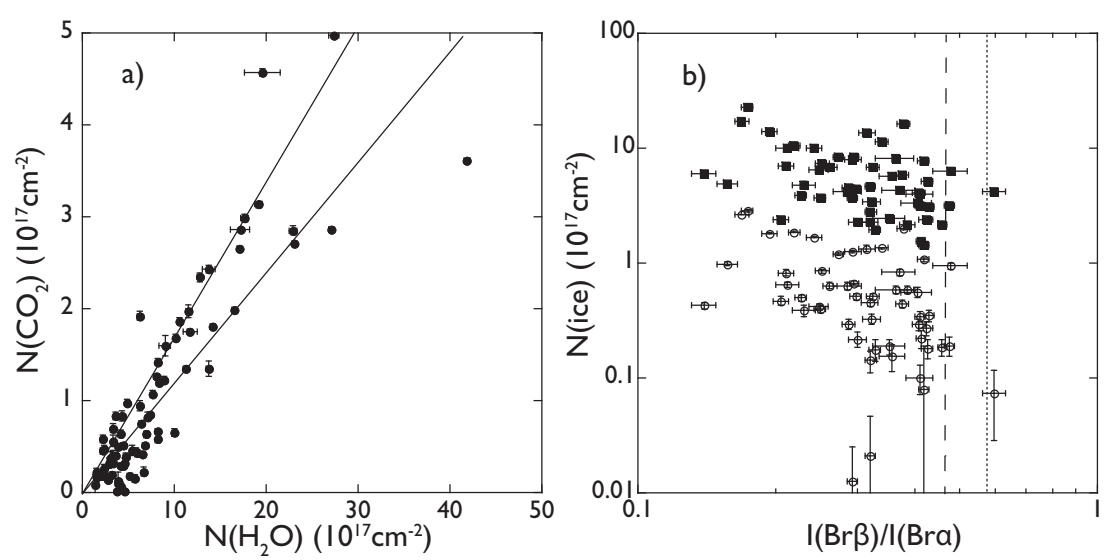

Figure 1. (a) Correlation of the column densities of $\mathrm{CO}_{2}$ and $\mathrm{H}_{2} \mathrm{O}$ ices for HII-PDR complexes observed with the IRC. The solid lines of the slopes of 0.17 and 0.12 are indicated as reference. The slope of 0.17 is suggested for Galactic massive YSOs in previous studies. (b) Correlations of the ice column densities and the ratio of $\operatorname{Br} \beta$ to $\operatorname{Br} \alpha$ line intensities. The filled squares show $\mathrm{H}_{2} \mathrm{O}$ ice, while the open circles indicate $\mathrm{CO}_{2}$ ice. The dotted line shows the ratio for Case B with the electron density of $10^{4} \mathrm{~cm}^{-3}$ and the temperature of $10^{4} \mathrm{~K}$. The dashed line suggests a possible threshold for the presence of the two ice species, which corresponds to $A_{v} \sim 5$.

indicate evidence for interstellar processing of ice species. Previous studies of the emission bands related to polycyclic aromatic hydrocarbons (PAHs) indicate that IRC spectra of those objects indeed show ice absorption features (Mori et al.2014; Onaka et al. 2014). Figure 1a shows the correlation of the column densities between $\mathrm{CO}_{2}$ and $\mathrm{H}_{2} \mathrm{O}$ ices for the present samples, which is in agreement with previous results for Galactic massive YSOs except for several data located below the correlation line(s), suggesting possible interstellar processing. Figure 1b plots the ice column densities against the HI line intensity ratio, which suggests a threshold for the presence of these ices. The suggested threshold is $A_{v} \sim 5 \pm 1$, being compatible with those found in the Taurus cloud (Whittet et al. 2001, 2007). The new IRC results support concurrent formation of $\mathrm{CO}_{2}$ and $\mathrm{H}_{2} \mathrm{O}$ ices and suggest common physical conditions present for ice formation in the ISM.

This work is based on observations of $A K A R I$, a JAXA project in participation of ESA. It is supported in part by a Grant-in-Aid from the JSPS (23244021).

\section{References}

Gibb, E. L., Whittet, D. C. B., Boogert, A. C. A., \& Tielens, A. G. G. M. 2004, ApJS, 151, 35 Ioppolo, S., van Boheemen, Y., Cuppen, H. M., et al. 2011, MNRAS, 413, 228

Mori, T. I., Onaka, T., Sakon, I., et al. 2014, ApJ, 744, 68

Noble, J., Fraser, H., Aikawa, Y., Pontoppidan, K. M., \& Sakon, I. 2013, ApJ, 775, 85

Oba, Y., Watanabe, N., Hama, T., et al. 2012, ApJ, 749, 67

Onaka, T., Matsuhara, H., Wada, T., et al. 2007, PASJ, 59, S401

Onaka, T., Mori, T. I., Sakon, I., et al. 2014, ApJ, 780, 114

Pontoppidan, K. M., Boogert, A. C. A., Fraser, H., et al. 2008, ApJ, 678, 1005

Shimonishi, T., Onaka, T., Kato, D., et al. 2008, ApJL, 686, L99

Shimonishi, T., Onaka, T., Kato, D., et al. 2010, A\&A, 514, A12

van Dishoeck, E. F. 2014, Faraday Discuss, 168, 9

Whittet, D. C. B., Gerakines, P. A., Hough, J. H., \& Shenoy, S. S. 2001, ApJ, 547, 872

Whittet, D. C. B., Shenoy, S. S., Bergin, E. A., et al. 2007, ApJ, 655, 332

Yamagishi, M., Kaneda, H., Ishihara, D., et al. 2015, ApJ, 807, 29 